\title{
Decision-making technology for changing the performance characteristic of the adaptive wheeled movers of complex robotic systems
}

\author{
Aleksey Ivanyuk \\ Vitaliy Karlov \\ Yuriy Serdobintsev \\ Department of Work Process Automation \\ Volgograd State Technical University \\ Volgograd, Russian Federation \\ ivanyuk_aleksei@mail.ru
}

\begin{abstract}
A solution is proposed for automating the process of controlling the wheel diameter of robotic systems and its main characteristics. Changes in rigidity, contact area with the surface, linear speed, turning radius contribute to higher mobility and increased maneuverability. A decision-making technology for changing the driving characteristics of adaptive propulsion systems has been developed with the focus on the probabilistic approach of estimating the faults of the positioning system modules in space.
\end{abstract}

Keywords - decision-making technology; adaptive propulsion systems; complex robotic systems; automated control system; strain measurement, probabilistic approach

Complex robotic systems (CRS) are utilized in industrial, agricultural, marine, military and live-saving purposes. The main types of propulsion systems are wheeled movers, walkers, caterpillars, anthropomorphic devices, etc. Ideas of using adaptive wheels able to change their rigidity, wheel size, contact area with the surface are increasingly being applied. Integrating adaptive wheels contributes to both higher mobility and increased maneuverability of wheeled movers; and integrating strain gauging enables the creation of automated systems that make decisions on changing the diameter of the wheel for the purpose of its most effective movement based on the geometry of obstacles. The automated system for controlling adaptive propulsion devices is able to reserve technological vision in case of their failures [1].

Adaptive propulsion systems (wheeled movers) are utilized in vehicles, in complex robotic systems for ground and space purposes, in marine robots used for the exploration and offshore production of hydrocarbons, as well as in seafloor mapping. Nowadays, seafloor exploration is considered one of the global tasks of high priority ranged from mineral exploration and seabed mining to pipe-laying at great depths. Wheeled robots are most often used in cartographic exploration. Complex seafloor landscapes and problems related to signal transmission prove the necessity of adaptive propulsion systems which can sense any obstacle and change the parameters of the wheel in order to get over this problem area without human aid. Operations of designing and creating adaptive wheels contribute to both higher mobility and increased maneuverability of wheeled movers [2].

The purpose of this study is to enhance the reliability of CRS's positioning control system. To accomplish the aforementioned purpose, the following tasks should be solved:

- to discuss possible causes of CRS positioning failures;

- to develop a life-cycle model in the form of a transition graph;

- to develop an algorithm for automatic control of the parameters of the wheel with regard to the landscape moved across;

- to develop a decision-making technology focused on changing the driving characteristics of CRS's adaptive propulsion systems.

To reveal any possible failure area of the CRS positioning system in space, let us consider the proposed design of the adaptive wheel (Fig.1) equipped with strain sensors for defining the stress-strain state of the elastic-metal body [3].

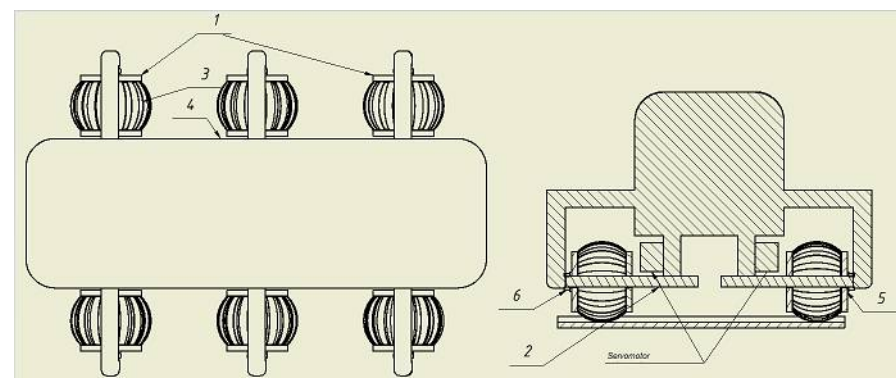

Fig.1. Virtual model for adaptive wheels of complex robotic system (lhs. - top view, rhs. - cross-sectional view)

The adaptive wheel comprises the following parts: $1-\mathrm{a}$ hub fixed to axle 2 that transmits driving torque to the wheels (each wheel is driven by its own servomotor); 3 - an elastic metal body made of elastic plates with strain sensors on their inner sides; 4 - a robot body; 5 - fixtures; 6 - outer hubs. Inner hubs located closer to the body are moved by a servomotor. 
Each wheel has its own actuator, which allows moving at a constant speed and in a straight line, for different diameters of the wheel (for example, when only one side of the robot is passing the obstacle). The calculator mechanisms are located on the hub inside the wheel [4].

To conduct the calculation and investigation procedures, it was assumed that the plates were absolutely elastic, the robot moved along a regular geometrically flat surface, and the obstacles were square-shaped steps; the coefficient of friction with the soil was recognized as constant [5].

The working principle of the adaptive wheel was as follows: bending resistors were fastened to each elastic wheel plate, on the inner sides. The resistors were bailed in such a way that the plate under no load would demonstrate resistance taken as the reference value, and the plate under load would have a smaller resistance. The sensor readings were transmitted to a microprocessor, which made a comparison and determined the location of the contact with the surface and other plates for the purpose of detecting the obstacle. If the resistance of the strain sensor on the plate decreased after contact with the ground, it would be necessary to increase the radius of the wheel because of the obstacle. The signal from the microprocessor was fed to the servomotor, which, in turn, by means of a gear transmission, moved the hubs from the inner side of the CRS [6].

Let's consider the block diagram for the CRS positioning system module failures (Fig.2).

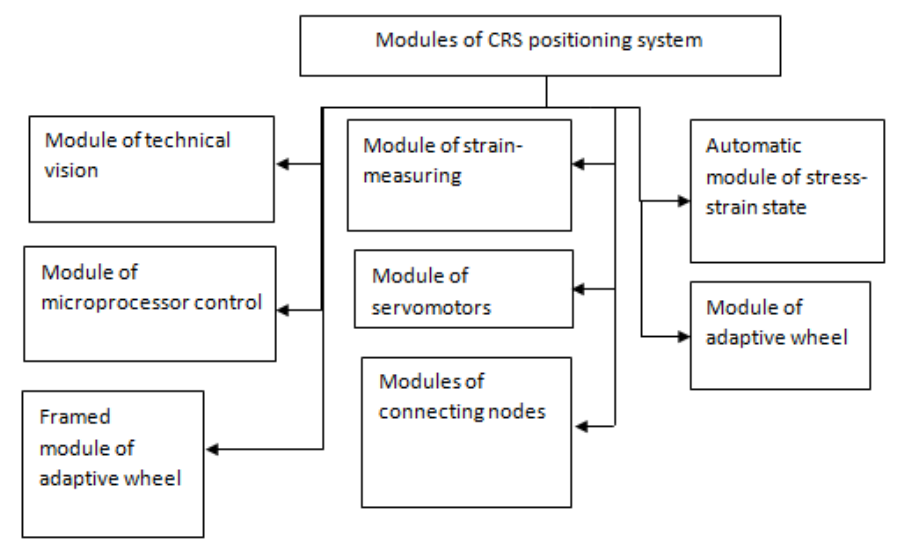

Fig. 2. Block diagram of failures of the CRS positioning system modules

To determine the probabilities of robotic system functioning in a particular phase (situation), a life-cycle model was used in the form of a transition graph (Fig. 3).

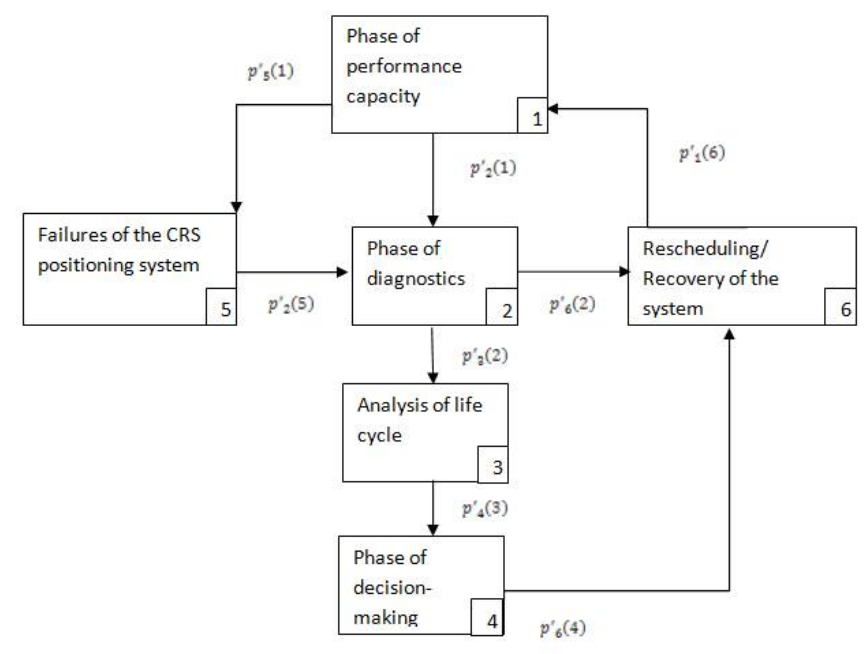

Fig.3. Life-cycle model in the form of a transition graph

The conditional probability of CRS functioning in the operational phase:

$$
P_{1}=\frac{p I_{1}(6)}{1+\sum T_{k=0}^{-1} p r_{k}(k-1)}
$$

Where $\mathrm{T}=6$ - total number of phases (situations);

- phase of diagnostics:

$$
P_{2}=\frac{p t_{2}(1)+p \prime_{2}(5)}{1+\sum_{k=0}^{T-1} P{ }_{k}(k-1)}
$$

- analysis of the life cycle of the CRS:

$$
P_{3}=\frac{p r_{3}(2)}{1+\Sigma T_{k=0}^{-1} P s_{k}(k-1)}
$$

- phase of decision-making:

$$
P_{4}=\frac{p s_{4}(3)}{1+\sum T_{k=0}^{-1} P / k(k-1)}
$$

- failures of the CRS positioning system:

$$
P_{5}=\frac{p I_{5}(1)}{1+\sum_{k=0}^{T-1} F f_{k}(k-1)}
$$

- rescheduling and recovery of the CRS positioning system:

$$
P_{6}=\frac{p_{0}(2)+p r_{0}(4)}{1+\sum \sum_{k=0}^{T-1} p r_{k}(k-1)}
$$

On the basis of possible failure points in the positioning system, a decision-making technology for changing the driving characteristics of the adaptive propulsion devices of robotic systems was developed (Fig. 4). 


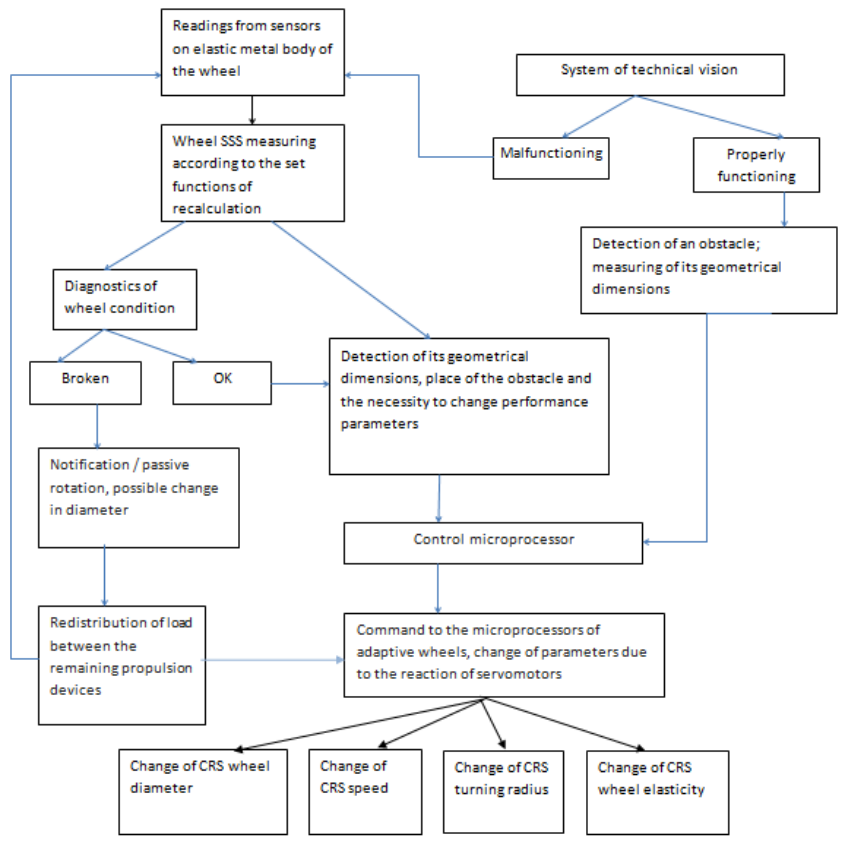

Fig.4. Decision-making technology for changing the driving characteristics of adaptive wheeled movers of robotic systems

Let's consider in more detail the technology of decisionmaking for changing the driving characteristics of adaptive propulsors of robotic systems. To determine the presence of obstacles, various systems of technical vision are used in robots. During the correct operation of these systems, when an obstacle is detected, both its dimensions and the distance to it are measured, and the information is sent to the control microprocessor. Afterwards, the command is sent to a certain servomotor of the adaptive wheel, in which the parameter is necessarily to be changed; e.g., to increase the diameter of the wheels on the right side of the CRS in order to get over the obstacle, without changing the direction.

In the event of a malfunction of the vision system or the impossibility of signal transmission, it is necessary for the CRS to automatically detect the presence of obstacles and to adjust the parameters of the adaptive propulsors. Because of a low speed of most of such robots, a solution was proposed for automatically determining the height of the obstacle due to the values obtained from the strain sensors of the adaptive wheel located on the elastic metal body.

According to the given functions, the values obtained by the sensors were recalculated to determine the SSS (stressstrain state) of the wheel frame.

In each module of automatic determination of the wheel SSS, two parallel processes occurred simultaneously: determination of both the presence of an obstacle and its dimensions, and diagnostics of the condition of the elastic metal body of the adaptive propulsor. During the first process, the wheel hit the obstacle; the elastic plates underwent deformation; and the calculation was done due to the values of the obstacle height obtained by the bending resistors, on the basis of which the control microprocessor, in accordance with the given functions, changed the elastic parameters of the wheel, its diameter or other parameters necessary to pass the obstacles.

Geometric calculations were done to determine the height of the obstacle (Fig.5), to verify the fulfillment of the condition for obstacle passing and to establish it necessary to change the diameter of the adaptive wheel of the CRS [7].

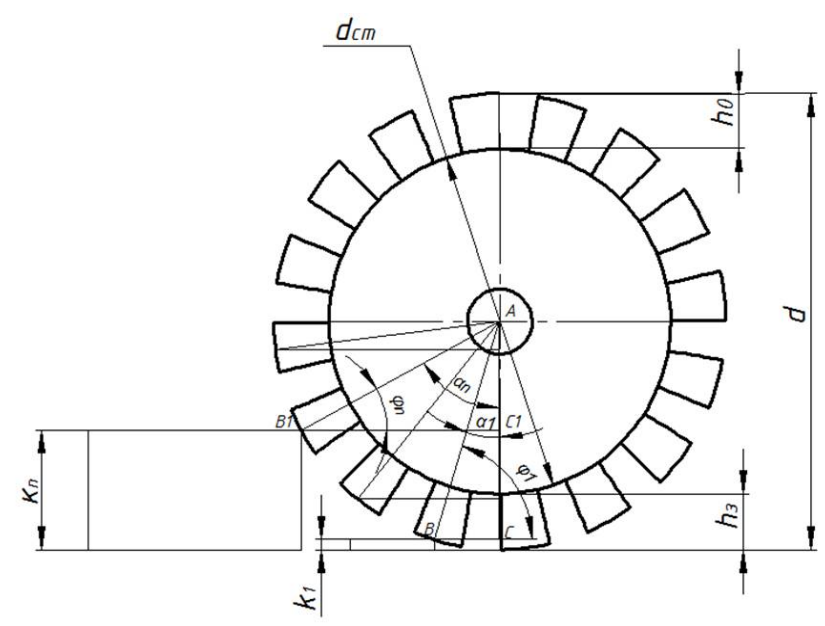

Fig.5. Geometry for calculating the system of adjustment of the diameter of the adaptive wheel in accordance with the height of the obstacle

Thus, if the function describing the dependence between strain-sensor resistance change and plate deformation is known, the calculation is done, as follows:

$$
\Delta h=f(\Delta c)
$$

This function can also be represented as a polynomial of "n" degree:

$$
h=a_{o}+a_{1} * c+a_{2} * c^{2}+\ldots+a_{n} * c^{n}
$$

Where $a_{n}$ - numerical coefficients; $c^{n}$ - values of resistance of the strain sensor.

Then, the following simple relation for a new diameter (which needs be changed) can be derived:

$$
d_{2}=d_{h}+h_{o}+h_{g}
$$

Where $d_{2}$ - new diameter;

- $d_{h}$-hub diameter;

- $h_{o}$ - height of elastic element under "zero" load;

- $h_{g}$ - height of elastic element at the point of contact with the ground.

Knowing the value by which the diameter should be increased, the function of transmitting the driving torque to the mechanism from the servomotor can be set. Calculations of wheel diameter adjustment are based on the following data.

Let " $n$ " be the number of elastic plates in the wheel, then the angle between them (the generators of the elastic metal body) will be the following: 


$$
\alpha_{1}=360 / \mathrm{n}
$$

Thus, the angle between the radius of the wheel and the contour line at the point of contact with the obstacle is:

$$
\varphi_{1}=180-\alpha_{1}-90
$$

Or in case of "collision with an obstacle" of the "b" plate:

$$
\varphi_{n}=90-b \alpha
$$

Due to the geometry of the triangle $\mathrm{AB} 1 \mathrm{C} 1$ :

$$
\sin \left(\varphi_{n}\right)=\frac{\left(\left(r+h_{3}\right)-k_{n}\right)}{r+h_{n}}
$$

Where $\mathrm{r}$ - radius of the hub (used for the convenience of calculations). Then, the height " $n$ " of the obstacle is calculated as:

$$
k_{n}=h_{3}+r\left(1-\sin \left(\varphi_{n}\right)\right)-h_{n} \sin \left(\varphi_{n}\right)
$$

The conditions of getting over an obstacle is:

$$
k_{n} \leq \frac{1}{2} d
$$

Then, the new minimum diameter is determined:

$$
d_{\text {new }}=2 k_{n}
$$

It should be noted that any change in diameter leads not only to increased maneuverability, but also to a decrease in the area of contact with the ground and, respectively, to the possibility of increasing the linear speed of movement and maneuverability by varying the turning radius of the robotic system [8].

During the diagnostics of the wheel, a constant comparison is made between the given reference deflection values, the elastic generators of the wheel frame and the obtained values from the sensors. If the deflection is long (above the critical norm), the time is also set by the operator, the microprocessor determines that the wheel is damaged, and it is necessary to redistribute the arrangement of the propellers to eliminate the displacement of the center of the CRS mass. Also, a warning about the damage of the propeller takes place and it is switched to passive rotation; extra reduction of the diameter is also possible to prevent a negative impact on neighboring wheels. The second reason for the smaller diameter of the damaged wheel is the removal of "the hooks" with the ground.

Diagnostics of the state of adaptive propulsors has a constant feedback, by which the constant control of the condition of CRS propellers is carried out and decisions are made in regard to any changes in its driving characteristics.

In order to ensure a reliable operation of the CRS, it is necessary to provide the announced working efficiency to its every module. In this case, working mode selection conditions can be considered with due regard to the probability of its functioning in this or that life-cycle phase. To increase the wear resistance and reliability of the most loaded moving parts (for example, coupling of servomotors, wheel shaft, bearings, etc.), it is proposed to use wear-resistant powder composite materials applied to the part by the method of gas-thermal coating. This allows extending the life cycle of the CRS.

\section{CONCLUSION}

The developed decision-making technology for changing the driving characteristics of adaptive propulsors, taking into account the functioning in different phases of the life cycle, makes it possible to increase the survivability and maneuverability of robotic systems of various types.

\section{REFERENCES}

[1] Yu.P. Serdobintsev, "Maintaining the efficiency of the working properties of technological equipment tribocoupling during the lifecycle: a Monograph", Volgograd, 2005, 353 p.

[2] Yu.P. Serdobintsev, "Deformation of Instrument Housings under External Pressure", Russian Engineering Research, vol. 37, No. 8, 2017, pp. 675-678.

[3] Yu.P. Serdobintsev, "Automated module for detecting deformation of bodies and their elements under external loads", Engineering Bulletin, vol. 5, 2017, pp. 38-41.

[4] A.K. Ivanyuk, Investigation of the strength and survivability of housings of electronic devices using the device for automated monitoring of stress-strain state, the $28^{\text {th }}$ International Innovation Conference of Young Scholars and Students (Moscow, December 2016), Proceedings, Moscow, 2017, pp. 30-33.

[5] Yu.P. Serdobintsev, "The use of strain gauges for changing the diameter of adaptive wheels of robotic systems", Advanced systems and control tasks, the $12^{\text {th }}$ All-Russian Scientific Conference and the $8^{\text {th }}$ Training School "Management and information processing in technical systems", Rostov-on-Don, 2017, pp. 558-565.

[6] A.K. Ivanyuk, M.V. Panchenko, V.A. Ryabchuk, Yu.P. Serdobintsev, P.M.172816 RF, MPK G01B7/16, G01N3/08, Device for measuring deformation during testing of structures for strength, VolgGTU, 2017.

[7] Yu.P. Serdobintsev, Analyzing the stress-strain state of object bodies subjected to hydrostatic pressure using modern CAD systems, the $2^{\text {nd }}$ International Conference on Industrial Engineering, Applications and Manufacturing (Chelyabinsk, Russia, 19-20 May 2016) 2016, DOI: 10.1109/ICIEAM.2016.7911678, p. 5

[8] A.K. Ivanyuk, Prototype of an automated device for monitoring the stress of deformed state of bodies experiencing external pressure, the 21 th Regional Conference of Young Scholars in Volgograd Region (Volgograd, 8-11 November 2016), VolgGTU, Voglograd, 2016, pp. 4547. 